\title{
HETEROGENEITY VERSUS THE COVID-19 PANDEMIC
}

Ramalingam Shanmugam ${ }^{1}$, Gerald Ledlow ${ }^{2}$ and Karan P. Singh ${ }^{3}$

${ }^{1}$ Professor of Statistics and Honorary Professor of International Studies

School of Health Administration, Texas State University

San Marcos, TX 78666, USA

Email: $\underline{\text { rs25@txstate.edu }}$

ORCID number 0000-0002-3388-1014

${ }^{2}$ Professor and Dean, Department of Healthcare Policy, Economics and Management

Email: gerald.ledlow@uthct.edu 


\section{Abstract}

21 In this paper, heterogeneity is formally defined, and its properties are explored. We define and

22 distinguish observable versus non-observable heterogeneity. It is proposed that heterogeneity among the

23 vulnerable is a significant factor in the contagion impact of COVID-19, as demonstrated with incidence

24 rates on a Diamond Princess Cruise ship in February 2020. Given the nature of the disease, its

25 heterogeneity and human social norms, pre-voyage and post-voyage quick testing procedures may

26 become the new standard for cruise ship passengers and crew. The technological advances in testing

27 available today would facilitate more humanistic treatment as compared to more archaic quarantine and

28 isolation practices for all onboard ship. With quick testing, identification of those infected and thus not

29 allowed to embark on a cruise or quarantining those disembarking and other mitigation strategies, the

30 popular cruise adventure could be available safely again. Whatever the procedures implemented, the

31 methodological purpose of this study should add valuable insight in the modeling of disease and

32 specifically, the COVID-19 virus.

34 Key Words: Observed homogeneity; non-observed homogeneity; over dispersion; under dispersion;

35 Poisson distribution; binomial distribution; Tango's test statistic.

\section{INTRODUCTION}

In the literature, the term heterogeneity echoes differently in various contexts. What is heterogeneity

39 or its antonym, homogeneity? Its root word lies in Greek "heterogenes" meaning different. In

40 epidemiology or statistics disciplines, the word heterogeneity is popularly commented to exist when the

41 variance is large. In insurance applications, for an example, the premium is assessed more if the insurer

42 is in a heterogeneous group with high hazard proneness (Spreeuw, 1999). Should a large (small) 
43 variance be indicative of heterogeneity (homogeneity)? Interesting discussions are given for

44 heterogeneity in Ecochard (2006); in healthcare disciplines, heterogeneity is referred to as different

45 outcomes among patients. Should the heterogeneity be connected to only a non-observable hidden trait

46 as done in genetics? Does heterogeneity refer dissimilar attributes across the subgroups of the population

47 itself even before sampling? Is heterogeneity really pointing to the non-identical nature in a random

48 sample or population? Should heterogeneity imply a shifting entity? In genetic studies, several authors

49 refer to genetic heterogeneity as rather too difficult to ascertain. What do they really mean? If alleles in

50 more than one locus exhibit susceptibility to a disease, there is a need to track the loci to infer their

51 heterogeneity. So, in a sense, the application of heterogeneity is really a discussion of an opposite of

52 similarity across loci. The reader is referred to Elston et al. (2003, pages 3404-344) for details. Hope and

53 Norris (2013) attempted to determine how heterogeneity played a role in judgements in the context of

54 crime victimization. Hence, what really is heterogeneity? A formal definition of heterogeneity is

55 constructed later in the article, then, its properties are explored and itemized.

However, in the epidemiology literature, using a random sample $y_{1}, y_{2}, \ldots, y_{n}$ from a population

whose main parameter is $\theta$, when the null hypothesis $H_{o}: \theta_{1}=\theta_{2}=\ldots=\theta_{n}$ is tested, it is named the

homogeneity test. This suggests that heterogeneity is really all about a shifting population. This creates

more confusion. Is the source of such confusion with respect to heterogeneity its ill communication? It is

evident that there is a lack of a clear definition of heterogeneity given by Hunink et al. (2018, Chapter

12) for details. Neither the Encyclopedia of Statistical Sciences nor the Encyclopedia of Biostatistics has even an entry, as if it is not pertinent in statistical disciplines. population is one of them, and the data should possess an under dispersion (i.e., variance of the binomial 
reflect equality between the mean and variance. When the main (incidence rate) parameter of a Poisson chance mechanism is stochastically transient, the unconditional observation of the random variable convolutes to an inverse binomial model (Ross, 2002). The inverse binomial distribution is known to attest that the variance is larger than its mean (Stuart and Ord, 2015, for details). Consequently, a comparison between the mean and variance characterizes only which type binomial, Poisson, or inverse binomial possesses the underlying chance mechanism we are sampling from but does not inform anything about heterogeneity.

With details about the probabilistic patterns among coronavirus confirmed, recovered, or cured individuals and those that succumb as fatalities/deaths in the thirty-two states/territories of India are given by Shanmugam (2020). To track the confusion with respect to heterogeneity, let us consider the data given in Table 1 (Mizumoto and Chowell, 2020), describing the spread of COVID-19 among the voyagers in a Diamond Princess Cruise ship, during the month of February 2020. The random variables $Y_{1}, Y_{2}$, and $Y_{3}$ denote, respectively, the number of COVID-19 cases, the number of asymptomatic cases and the number of symptomatic cases among them in time (date). Under a given COVID-19's prevalence rate, $\lambda>0$, the number $Y_{1}$ perhaps follows a Poisson probability pattern. For a given number of COVID-19 cases in a date, the number $Y_{2}$ perhaps follows a binomial probability pattern with parameters $\left(y_{1}, p\right)$, where $0<p<1$ denotes the chance for a COVID-19 case to exhibit no symptoms. Naturally, the number $Y_{3}$ should follow a binomial probability pattern with parameters $\left(y_{1}, 1-p\right)$. There is an implicitness between $Y_{2}$ and $Y_{3}$, in the sense that $Y_{2}+Y_{3}=Y_{1}$. There are three-time oriented groups of COVID-19 incidences in Table 1. Is there an observable heterogeneity among the three groups? If so, is it due to a non-observable (parametric) heterogeneity? How do we define and distinguish observable versus non-observable heterogeneity? A literature search in epidemiology and/or biostatistics does not provide an answer to this question. 
It is evident that the average of COVID-19 cases is an estimate of COVID-19's prevalence rate (i.e.,

$\hat{\lambda}$ in Table 1). Their estimates impress that the prevalence rate is transient, not constant across every pair

91 of two-day duration dyads. The Poisson population from which the COVID-19 cases are drawn ought to

92 have been dynamic, implying the existence of a Poisson heterogeneity. How do we define and/or capture

93 the heterogeneity level? This is the theme and purpose in this research article. asymptomatic cases, $y_{2}$ and the remaining are symptomatic cases, $y_{3}$. That is, $y_{2}$ and $y_{3}$ are complementary but $y_{2}+y_{3}=y_{1}$. Is there heterogeneity in each of the two sub-binomial populations, whether there is a heterogeneity in $y_{1}$ ? How should each binomial heterogeneity be defined and computed? In other words, is binomial heterogeneity different from that of Poisson heterogeneity? If so, what are the differences? A literature search in epidemiology and/or biostatistics offers no help to prove either the existence or absence of binomial heterogeneity in the data for $y_{2}$ or $y_{3}$ in Table 1 . Hence, we continue probing matters with respect to heterogeneity. heterogeneity, and that is exactly what this article is trying to accomplish. Hence, we first define and construct an approach for the idea of heterogeneity. To be specific, we first discuss Poisson heterogeneity and then take up binomial heterogeneity. Maybe our research direction about heterogeneity is, perhaps, pioneering. However, we believe that our approach is easily extendable for many other similar methodological setups. We illustrate our definition and all derived expressions for

109 heterogeneity using COVID-19's data pertaining to the Diamond Princess Cruise ship, Yokohama, 2020 110 as displayed in Table 1. 


\section{POISSON AND BIONOMIAL HETEROGENEITIES}

Applied epidemiologists emphasize that heterogeneity is of paramount importance in extracting and

114 interpreting data evidence. Many data analysts are convinced that an unrecognized heterogeneity leads

115 to a biased inference. To begin with, what is heterogeneity? It is a factor causing non-similarities. If so,

116 how many sources are there? We contemplate that there are two sources for heterogeneity to exist. One

117 source ought to be from the drawn random sample of observations: $y_{1}, y_{2}, \ldots ., y_{n}$, which we recognize as

118 observable heterogeneity. Would the sampling variability, $\operatorname{Var}\left[f\left(y_{1}, y_{2}, \ldots ., y_{n} \mid \theta\right)\right]$ for a selected statistic

$119 f\left(y_{1}, y_{2}, \ldots ., y_{n} \mid \theta\right)$ express the observable heterogeneity? Another source is manifested in non-

120 observable parameter, $\theta$ of the chance mechanism, which we recognize as non-observable

121 heterogeneity. Would a non-uniform stochastic pattern of $\theta$ be indicative of the non-observable

122 homogeneity? If the chance mechanism perversely selects a probability density function (pdf) for $\theta$,

123 how would it manifest itself to portray the non-observable heterogeneity? Both observable and non-

124 observable heterogeneity together ought to be involved to make any definition of heterogeneity

125 complete. If so, how do we integrate them? Often, under/over-dispersion is confused with heterogeneity.

126 It seems that the over/under dispersion is precipitated by heterogeneity but not the other way. It is not

127 obvious or proven so far in the epidemiology literature on whether the converse is true. We focus only

128 on Poisson and binomial populations to address heterogeneity, and these arguments can be repeated for

129 other populations considering similar methods.

\subsection{POISSON HETEROGENEITY}

132 Recall that the random integer, $Y_{1}$ denoting the number of COVID-19 cases in a place (like the

133 Diamond Princess cruise ship) at a time (like February, 2020) is a Poisson random variable with a

134 specified prevalence rate, $\lambda>0$. That is, the conditional probability of observing $y_{1}$ number of COVID- 
13519 cases under a prevalence rate $\lambda>0$ is $\operatorname{Pr}\left[Y_{1}=y_{1} \mid \lambda\right]=e^{-\lambda} \lambda^{y_{1}} / y_{1} ! ; y_{1}=0,1,2, \ldots ; \lambda>0$ with its

136 expected number $E\left[Y_{1} \mid \lambda\right]=\lambda$ and variability $\operatorname{Var}\left[Y_{1} \mid \lambda\right]=E\left[Y_{1} \mid \lambda\right]$. The reader is referred to Rajan and

137 Shanmugam (2020) for detailed derivations of the Poisson mean and variance. The prevalence parameter

$138 \lambda$ itself is crucial in our discussions. The Poisson variability cannot be heterogeneity, because the

139 expected value also changes when the variability changes due to their inter-relatedness. Realize that no

140 two individuals on the ship are assumed to have the same level of susceptibility to the COVID-19 virus.

141 It is reasonable to imagine that the prevalence levels follow a conjugate, stochastic gamma distribution.

142 The so-called conjugate prior knowledge in the Bayesian framework smooths the statistical analytic

143 process. It is known that the conjugate prior for the Poisson distribution is gamma, whose pdf is

$$
c(\lambda \mid \alpha, \beta) d \lambda=e^{-(\alpha \lambda)}(\alpha \lambda)^{\beta-1} d(\alpha \lambda) / \Gamma(\beta) ; \alpha>0 ; \beta>0,
$$

with an average. $E(\lambda \mid \alpha, \beta)=\frac{\beta}{\alpha}$ and variability $\operatorname{Var}(\lambda \mid \alpha, \beta)=E(\lambda \mid \alpha, \beta) / \alpha$, where the parameters $\alpha$ and $\alpha>0$ causes the variability in the COVID-19's prevalence rate to fluctuate up or down, and, hence, you would anticipate the heterogeneity to involve the hyperparameter $\alpha$. But the question is how?

We assume that the probability of observing a non-negative COVID-19 case, $y_{1}$ is a Poisson under a stable sampling population $\operatorname{Pr}\left(Y_{1} \mid \lambda\right)$ with an expected number $E\left(Y_{1} \mid \lambda\right)=\lambda$ and a variability

$153 \operatorname{Var}\left(Y_{1} \mid \lambda\right)=E\left(Y_{1} \mid \lambda\right)$. With replications, the observable heterogeneity should become estimable. That is

154 to mention, the maximum likelihood estimate (MLE) of the COVID-19 prevalence rate is the average 155 number, $\bar{y}_{1}$, of the observations. To discuss the non-observable heterogeneity, we need to integrate its 
conjugate prior $c(\lambda \mid \alpha, \beta)$ for the non-observable $\lambda$ with the likelihood $\operatorname{Pr}\left(Y_{1} \mid \lambda\right)$ and it results in an update

and it is called posterior pdf for $\lambda$. The expressions for non-observable heterogeneity, observable

\subsection{BINOMIAL HETEROGENEITY}

In this section, we explore heterogeneity for two sub-binomial processes emanating from a Poisson

161 process. The asymptomatic number, $Y_{2}$ and symptomatic number, $Y_{3}$ of COVID-19 cases are two

162 branching binomial random numbers out of the Poisson random number, $Y_{1}=0,1,2, \ldots$; of COVID-19

163 cases. These two split random variables are complementary of each other in the sense that $Y_{2}+Y_{3}=Y_{1}$.

164 Then, what are the underlying model for $Y_{2}$ and for $Y_{3}$ ? Are they correlated random variables? If so, what

165 is their correlation? These are pursued in this section.

166 Let $I$ be an indicator random variable defined as: $I_{i}=1$ for a COVID-19 case to be asymptomatic

167 with a probability, $0<p<1$ and $I_{i}=0$ for the case to be symptomatic with a probability, $0<1-p<1$.

168 Then, for a fixed $y_{1}$, the random variable, $Y_{2}=\sum_{i=1}^{Y_{1}} I_{i}$ follows a binomial probability distribution with

169 parameters $\left(y_{1}, p\right)$. Likewise, for a fixed $y_{1}$, the random variable, $Y_{3}=y_{1}-Y_{2}$ follows a complementary

170 binomial distribution with parameters $\left(y_{1}, 1-p\right)$. That is,

$\operatorname{Pr}\left(Y_{2}=y_{2} \mid y_{1}, p\right)=\left(\begin{array}{l}y_{1} \\ y_{2}\end{array}\right) p^{y_{2}}(1-p)^{y_{1}-y_{2}} ; y_{2}=0,1,2, \ldots \ldots, y_{1} ; 0<p<1$

172 and

$\operatorname{Pr}\left(Y_{3}=y_{3} \mid y_{1}, p\right)=\left(\begin{array}{l}y_{1} \\ y_{3}\end{array}\right)(1-p)^{y_{3}} p^{y_{1}-y_{3}} ; y_{3}=0,1,2, \ldots \ldots, y_{1} ; 0<1-p<1$ are given in Appendix II. 


\section{TANGO INDEX}

Lastly, we develop the Tango index and its significance level over the time period. Tango (1984)

proposed an index to detect disease clusters in grouped data. This index received considerable attention

$$
A_{3 \times 3}=\left(\begin{array}{ccc}
-1 & 0 & 1 \\
1 & -1 & 0 \\
0 & 1 & 0
\end{array}\right),
$$

where the third column of the matrix needs no explanation. The Tango's statistic $T=\underline{r}^{\prime} A \underline{r}$ follows a chi- 
In this section we illustrate all the concepts and expressions of Section 2. Let us consider the

198 COVID-19 data in Table 1 for the Diamond Princess Cruise Ship, 2020. The Diamond Princess is

199 a cruise ship registered in Britain and operated across the globe. During a cruise that began on 20

200 January 2020, positive cases of COVID-19 linked to the pandemic were confirmed on the ship in

201 February 2020. Over 700 people out of 3,711 became infected (567 out of 2,666 passengers and 145 out

202 of 1,045 crew), and 14 passengers died. To be specific, on the $15^{\text {th }}$ of February 2020, 67 people were

203 infected, on the $16^{\text {th }}$ of February 2020, 70 people were infected, on the $17^{\text {th }}$ of February 2020 , there were

20499 COVID-19 cases, on the $18^{\text {th }}$ of February, another 88 cases were confirmed. The U.S. government

205 initially asked Japan to keep the passengers and crew members on board the ship for 14 days. The U.S.

206 government, however, later decided to bring them to an Air Force base in California and a base in San

207 Antonio, Texas.

208 For each specified day in the first column in Table 1, the estimate of COVID-19's prevalence rate

209 and its variance are calculated using expressions $\hat{\lambda}=\bar{y}_{1}$ and $\operatorname{Var}\left(Y_{1} \mid \lambda\right)=s_{y_{1}}^{2}$. Both the prevalence and its

210 variability increased and then decreased over the days. However, their correlation, $\hat{\rho}_{Y_{2}, Y_{3}}$ is calculated

211 using the observed numbers on $y_{2}$ and $y_{3}$ for each day (see in Table 2) and the estimated correlations had

212 been stable over the days. Substituting $\hat{\lambda}=\bar{y}_{1}$ and $\operatorname{Var} r\left(Y_{1} \mid \lambda\right)=s_{y_{1}}^{2}$ in the expression

213

$$
\hat{H}_{\lambda}=\frac{\hat{\lambda}}{\hat{\lambda}+\operatorname{Var}\left(Y_{1} \mid \lambda\right)}
$$

214 we obtained the non-observable heterogeneity and displayed in Table 2. The non-observable Poisson

215 heterogeneity for $y_{1}$ was high on the beginning day, came down later, and then increased. Using $\hat{\lambda}=\bar{y}_{1}$ and

$216 \operatorname{Var}\left(Y_{1} \mid \lambda\right)=s_{y_{1}}^{2}$ in the expression 
$\hat{H}_{\bar{y}_{1}}=\left[1+\frac{\left(1+\frac{\bar{\lambda}}{s_{\lambda}^{2}}\right)}{6}\right]^{-1}$

218 we obtained the observable heterogeneity and displayed in Table 2. The observable Poisson

219 heterogeneity was low on the first day, increased and then decreased. Note in Table 2 that the observable

220 and non-observable Poisson heterogeneities are inversely proportional. In other words, the estimate of

221 the shape and scale parameter in the Bayesian approach are respectively $\hat{\alpha}=\frac{\bar{\lambda}^{2}}{s_{\lambda}^{2}}$ and $\hat{\beta}=\frac{\bar{\lambda}}{s_{\lambda}^{2}}$ (see their

222 values in Table 2). The shape parameter value decreased consistently over the days. The scale parameter

223 was high to begin with, then increased later. The distance, $d\left(y_{1}, \lambda\right)$ between the observable and non-

224 observable Poisson mechanism for $y_{1}$ is calculated using the expression

$$
d\left(y_{1}, \lambda\right)=\{\beta(1-\beta) \pm 1\}\left(\frac{\alpha}{\{1+\beta\}^{2}}\right)
$$

226 and displayed in Table 2. Notice that the distance was large to begin with, then decreased but increased

227 later over the days.

228 Note that we compute $\hat{p}_{i}=\frac{y_{2}}{y_{1}}$ for the $i^{\text {th }}$ day. Then, we calculate the

$$
\text { average: } \quad \bar{p}=\sum_{i=1}^{2} p_{i} / 2 \text { and the variance: } s_{p}^{2}=\frac{\left(p_{1}-p_{2}\right)^{2}}{4} \quad(\hat{p} \text { in Table } 1),
$$

230 and it had been steadily increasing over the days since $15^{\text {th }}$ February 2020 . This is something valuable for 231 medical professionals learning the clinical nature of COVID-19. Using the expression,

$$
o d d s_{Y_{2}} \approx e^{-p \lambda}\left\{1+e^{-p(1-p) \lambda}\right\}
$$

233 in Section 2.2, we calculated the odds for a COVID-19 case to become an asymptomatic type and 234 displayed in Table 2. 
Likewise, using the expression

$$
\operatorname{odds}_{Y_{3}} \approx e^{-(1-p) \lambda}\left\{1+e^{-p(1-p) \lambda}\right\}
$$

we estimated the odds for a COVID-19 case to become a symptomatic case as shown in Table 2 . Notice that both odds ( $O d d s_{Y_{2}}$ and $\left.O d d s_{Y_{3}}\right)$ are low but their odds ratio,

$$
O R_{\frac{Y_{3}}{Y_{2}}}=e^{-(1-2 p) \lambda}
$$

is not negligible but reveals that the situation is favorable to symptomatic rather than asymptomatic.

This discovery is feasible because of the approach, and it is an eye-opening reality for the medical

professionals in their desire to control the spread of the COVID-19 virus. Both the observable, $\hat{H}_{y_{2} \mid y_{1}}$ and non-observable, $\hat{H}_{y_{1}, \gamma, \delta}$ binomial heterogeneity (see their values in Table 3 ) were decreasing for the number, $y_{2}$ of asymptomatic COVID-19 cases. The distance, $d\left(y_{2}, p\right)$ between the observable and nonobservable for asymptomatic cases was moderate in the beginning, then increased, and then decreased over the next days (see their values in Table 3). However, the distance, $d\left(Y_{2}, Y_{3}\right)$ between the observable, $y_{2}$ of the asymptomatic cases and the observable, $y_{3}$ of the symptomatic cases was narrow, then wider, and then moderate over the days (their values in Table 3).

For a COVID-19 case to become a symptomatic type, the chance is moderate to less and then more over the days $(1-\bar{p}$ in Table 3$)$. The estimate of the shape and scale parameter happened to be $\hat{\gamma}$ and $\hat{\delta}$ respectively (see their values in Table 3). Both the shape parameter and the scale parameter values decreased drastically over the days. From the p-values in Table 4, we infer that the prevalence rate, $\hat{\lambda}$, the distances, $d\left(y_{1}, \lambda\right), d\left(y_{2}, p\right)$ and $d\left(Y_{2}, Y_{3}\right)$ do differ significantly over the three groups of dyad days. The chance for COVID-19 to become an asymptomatic type does not differ significantly across the three groups. On the contrary, the non-observable heterogeneities $H_{\hat{\lambda}}$ of the Poisson random number, $y_{1}$ and 
$257 \hat{H}_{y_{1}, \gamma, \delta}$ of the binomial random number, $y_{2}$ are not significant. Likewise, the observable heterogeneities $\hat{H}_{y_{1}}$

258 of the Poisson random number, $y_{1}$ and $\hat{H}_{y_{2} \mid y_{1}}$ of the binomial random number, $y_{2}$ for a given $y_{1}$ are not

259 significant.

260

261 Table 1. COVID-19 in Cruise Ship, 2020, Mizumoto et al. (2020)

\begin{tabular}{|l|l|l|l|l|l|l|l|}
\hline Date & $Y_{3}$ & $Y_{2}$ & $Y_{1}$ & $\bar{\lambda}=\bar{y}_{1}$ & $s_{\lambda}^{2}=\operatorname{Var}\left(Y_{1}\right)$ & $\begin{array}{c}O_{\frac{1 \rightarrow 2}{0 \rightarrow 1}} \\
\text { Odd }\end{array}$ & \\
\hline Feb 15-16, 2020 & 29,32 & 38,38 & 67,70 & 68.5 & 4.5 & 0.5001 & $1.7 \mathrm{E}-30$ \\
\hline Feb 17-18, 2020 & 29,23 & 70,65 & 99,88 & 93.5 & 60.5 & 0.5000 & $2.4 \mathrm{E}-41$ \\
\hline Feb 19-20, 2020 & 11,7 & 68,6 & 79,13 & 46 & 21.78 & 0.5002 & $1.0 \mathrm{E}-20$ \\
\hline
\end{tabular}

Table 2. Results for Mizumoto et al.'s COVID-19 Data in Diamond Princess

\begin{tabular}{|c|c|c|c|c|c|c|}
\hline Date & $O R_{\frac{Y_{3}}{Y_{2}}}$ & $\hat{H}_{y_{1}}$ & $\hat{\beta}$ & $\hat{\alpha}$ & $d\left(y_{1}, \lambda\right)$ & $H_{\hat{\lambda}}$ \\
\hline 15,16 Feb 2020 & 943.88 & 0.27 & 15.22 & 1042.72 & 857.81 & 0.93 \\
\hline 17,18 Feb 2020 & $7.36 \mathrm{E}+17$ & 0.70 & 1.54 & 144.50 & 18.79 & 0.61 \\
\hline 19,20 Feb 2020 & $9.69 \mathrm{E}+11$ & 0.65 & 2.11 & 97.15 & 23.56 & 0.67 \\
\hline
\end{tabular}

265 Table 3. Results for Asymptomatic COVID-19 Cases in Mizumoto et al. (2020)

\begin{tabular}{|l|l|l|l|l|l|l|}
\hline Date & $\begin{array}{l}1-\bar{p}= \\
1-\operatorname{Ave}\left(\frac{y_{2}}{y_{1}}\right)\end{array}$ & $\begin{array}{l}s_{p}^{2} \\
=\operatorname{Var}\left(\frac{y_{2}}{y_{1}}\right)\end{array}$ & $\hat{H}_{y_{1}, \gamma, \delta}$ & $\hat{H}_{y_{2} \mid y_{1}}$ & $d\left(y_{2}, p\right)$ & $d\left(Y_{2}, Y_{3}\right)$ \\
\hline 15,16 Feb 2020 & 0.45 & 0.0002 & $\mathbf{0 . 9 9}$ & $\mathbf{0 . 9 5}$ & 37.125 & 6.85 \\
\hline
\end{tabular}




\begin{tabular}{|l|l|l|l|l|l|l|}
\hline 17,18 Feb 2020 & 0.28 & 0.0004 & $\mathbf{0 . 9 8}$ & $\mathbf{0 . 8 9}$ & 66.6 & 41.14 \\
\hline 19,20 Feb 2020 & 0.34 & 0.0796 & 0.10 & $\mathbf{0 . 7 4}$ & 29.7 & 14.72 \\
\hline
\end{tabular}

Table 4. Tango's Test Statistic and Its P-Value for Several Entities

\begin{tabular}{|l|l|l|l|l|l|l|l|l|}
\hline Tango & $\hat{H}_{y_{1}}$ & $H_{\hat{\lambda}}$ & $\bar{p}$ & $\hat{H}_{y_{1}, \gamma, \delta}$ & $\hat{H}_{y_{2} \mid y_{1}}$ & $d\left(y_{1}, \lambda\right)$ & $d\left(y_{2}, p\right)$ & $d\left(Y_{2}, Y_{3}\right)$ \\
\hline Ttatistic & & & & & & & \\
\hline p-value & 0.87 & 0.83 & 0.81 & $\mathbf{0 . 6 7}$ & $\mathbf{0 . 7 7}$ & $0.0 \mathrm{E} 100$ & $2.4 \mathrm{E}-57$ & $7.5 \mathrm{E}-164$ \\
\hline
\end{tabular}

\section{DISCUSSION AND CONCLUSION}

The risk of contracting the COVID-19 virus during a cruise is more than in a community setting, as confined spaces discourage non-pharmaceutical mitigation strategies such as social distancing to be weakly implemented and breathing air is tightly internalized. More nations are afraid to let the voyagers come ashore at the seaports. Ships are not even permitted to dock at the port, as to not complicate virus mitigation efforts by the local surrounding communities. The scenario seems to be anti-humanistic. The medical doctors and/or pharmaceutical service were strained due to the infected and COVID-19-free voyagers. Lack of clear symptoms among those that were infected added to difficulties in managing the dispose of the COVID-19 fatalities (bodies), in a safe manner? 
283 dealing with the intention of reducing the spread of COVID-19 if not its total control. Still much of

284 COVID-19 is a mysterious pandemic. It is clear that non-pharmaceutical mitigation strategies such as

285 social distancing, utilization of face coverings, frequent hand sanitization, infected people quarantining

286 on board, and severely controlled ship cleanliness and sanitation standards are required; this may only be

287 successful with limited numbers of passenger and crew members. Given the nature of the disease, its

288 heterogeneity and human social norms, pre-voyage and post-voyage quick testing procedures may

289 become the new standard for cruise ship passengers and crew. The technological advances in testing

290 provided today would facilitate more humanistic treatment as compared to more archaic quarantine and

291 isolation practices for all onboard ship. With quick testing, identification of those infected and thus not

292 allowed to embark on a cruise or quarantine those disembarking, and other mitigation strategies, the

293 popular cruise adventure could be available safely again. Whatever the procedures implemented, the

294 methodological purpose of this study should add valuable insight in the modeling of disease and

295 specifically, the COVID-19 virus.

297 Funding

298 Conflict of interest

299

300

301

302

303

304

305
None.

The authors have no conflict of interest.

Availability of data and materials There is no other data or materials other than what are in the manuscript itself.

Code availability None.

Authors' contribution

The authors contributed everything in the manuscript.

Acknowledgements

The authors thanks Texas State University and The University of

Texas Health Science Center at Tyler for the support to write this article. 


\section{REFERENCES}

1. Blumenfeld D. Operations Research Calculations Handbook, CRC Press, Boca Raton, Florida, 2020.

2. Ecochard J. Heterogeneity in fecundability studies: issues and modeling, Statistical Methods in Medical Research; 2006, 15: 141-160.

3. Elston R, Olson J, Palmer L. Biostatistical Genetics and Genetic Epidemiology, Wiley Press, Baffins Lane, Chichester, West Sussex, UK, 2002.

4. Hope T, Norris P. Heterogeneity in the frequency distribution of crime victimization, Journal of Quantitative Criminology; 2013, 29 (4): 543-576.

5. Hunink MG, Weinstein MC, Wittenberg E, Drummond MF, Pliskin JS, Wong JB, Glasziou PF. Decision making in health and medicine: Integrating evidence and values, Cambridge University Press, Cambridge, UK, 2018. Management; 2016, 2 (6): 70-79.

6. Khokhlov V. Conditional value-at-risk for elliptical distributions, Evropský časopis Ekonomiky a

7. Mizumoto K, Chowell G. Transmission potential of the novel coronavirus (COVID-19) onboard the Diamond Princess Cruises Ship, 2020, Infectious Disease Modelling; 2020, 5: 264-270.

8. Mizumoto K, Kagaya K, Zarebski A, Chowell G. Estimating the asymptomatic proportion of coronavirus disease 2019 (COVID-19), cases on board the Diamond Princess cruise ship, Yokohama, 2020. Euro Surveill;2020, 25(10): 1560-7917.

9. Rajan C, Shanmugam R. Discrete Distributions in Engineering and the Applied Sciences, Synthesis Lectures on Mathematics and Statistics, Vol. 12, Pages 1-227, Morgan \& Claypool 
10. Ross S. (2002) A First Course in Probability, sixth edition, Prentice Hall, Upper Saddle River, New Jersey 07458, 2002.

11. Shanmugam R. Probabilistic Patterns among Coronavirus Confirmed, Cured and Deaths in Thirty-two India's States/Territories, International Journal of Ecological Economics and Statistics, 41 (4), 45-56, 2020, in press.

12. Shanmugam R, Radhakrishnan R. Incidence jump rate reveals over/under dispersion in count data, International Journal of Data Analysis, and Information Systems, 3(1), 1-8, 2011.

13. Spreeuw J. Heterogeneity in hazard rates in insurance, Tinbergen Institute of Research Series, 210, Ph. D. thesis, University of Amsterdam, Amsterdam, 1999.

14. Stuart A, Ord K. Kendall's Advanced Theory of Statistics, Volume 1. Oxford University Press, London, 2015. 


\section{APPENDIX I}

\section{2}

353

354

355

356

357

358

359

360

361

362

363

364

365

366

367

368

\section{Poisson Heterogeneity: Derivations}

It is known that the conjugate prior for the Poisson distribution is gamma, whose pdf is

$$
c(\lambda \mid \alpha, \beta) d \lambda=e^{-(\alpha \lambda)}(\alpha \lambda)^{\beta-1} d(\alpha \lambda) / \Gamma(\beta) ; \alpha>0 ; \beta>0
$$

with an average. $E(\lambda \mid \alpha, \beta)=\frac{\beta}{\alpha}$ and variability $\operatorname{Var}(\lambda \mid \alpha, \beta)=E(\lambda \mid \alpha, \beta) / \alpha$, where the parameters $\alpha$ and $\beta$ are recognized as hyper-parameters (Rajan and Shanmugam, 2020). Notice that the hyper

parameter $\alpha>0$ causes the variability in the COVID-19's prevalence rate to fluctuate up or down and hence, you would anticipate the heterogeneity to involve the hyperparameter $\alpha$.

$$
c\left(\lambda \mid y_{1}, \alpha, \beta\right)=\operatorname{Pr}\left(y_{1} \mid \lambda\right) c(\lambda \mid \alpha, \beta) / \int_{-\infty}^{\infty} \operatorname{Pr}\left(y_{1} \mid \lambda\right) c(\lambda \mid \alpha, \beta) d \lambda
$$

is the posterior pdf of the non-observable $\lambda$. Also, the denominator

$$
\int_{0}^{\infty} \operatorname{Pr}\left(y_{1} \mid \lambda\right) c(\lambda \mid \alpha, \beta) d \lambda=\Gamma\left(\alpha+y_{1}\right) /(1+\beta)^{\alpha+y_{1}}
$$

in a Bayesian framework, is called the marginal distribution. With $\Delta_{\lambda}=\lambda-E(\lambda)$, it is clear that

$\int_{-\infty}^{\infty} \Delta_{\lambda} c(\lambda \mid \alpha, \beta) d \lambda=0$, note that the prior variance is

$$
\operatorname{Var}(\lambda \mid \alpha, \beta)=\int_{-\infty}^{\infty} \Delta_{\lambda}^{2} c(\lambda \mid \alpha, \beta) d \lambda
$$

Because the prior is conjugate, its counterpart's variability

$$
\operatorname{Var}\left(\lambda \mid y_{1}, \alpha, \beta\right)=\int_{-\infty}^{\infty}\left(\lambda-E\left[\lambda \mid y_{1}, \alpha, \beta\right)^{2} c(\lambda \mid \alpha, \beta) d \lambda\right.
$$


369 is minimal when the Bayes estimate of the non-observable is the posterior mean, $\lambda_{\text {Bayes }}=E\left[\lambda \mid y_{1} \alpha, \beta\right]$,

370 where

$$
E\left[\lambda \mid y_{1}, \alpha, \beta\right]=\frac{\left(\alpha+\bar{y}_{1}\right)}{\beta}
$$

Differentiating the log-likelihood function

$$
\ln L\left(n \bar{y}_{1}, \lambda\right)=n \bar{y}_{1} \ln \lambda-n \lambda+\sum_{i=1}^{n} \ln \left(y_{i} !\right)
$$

374 with respect to the non-observable parameter, $\lambda$, setting it equal to zero and solving it, we obtain the

375 MLE and it is $\hat{\lambda}_{m l e}=\bar{y}_{1}$. Because of the invariance property of the MLE, it is involved. The invariance 376 property refers to that the MLE of a function of the parameter is the function of the MLE of the 377 parameter. Also, it is known (Blumenfeld, 2010) that

382 Hence, we are ready now to define the non-observable heterogeneity below in the Definition 1.

$$
E_{\text {prior }} E_{\text {ikelihood }}\left(\bar{y}_{1} \mid \lambda\right)=E\left(\bar{y}_{1}\right) \quad \text { and }
$$

$$
\operatorname{Var}\left(\bar{y}_{1}\right)=E_{\text {prior }} \operatorname{Var}_{\text {likelihood }}\left(\bar{y}_{1} \mid \lambda\right)+\operatorname{Var}_{\text {prior }} E_{\text {likelihood }}\left(\bar{y}_{1} \mid \lambda\right)
$$

$$
H_{\lambda}=\left[1+\frac{\text { Var }_{\text {prior }} E_{\text {likelihood }}\left(\bar{y}_{1} \mid \lambda\right)}{E_{\text {prior }} \operatorname{Var}_{\text {likelihood }}\left(\bar{y}_{1} \mid \lambda\right)}\right]^{-1} \in[0,1] .
$$

388 Following the Definition 1, we obtain the non-observable heterogeneity of the COVID-19 cases is 


$$
H_{\lambda}=\left[1+\frac{1}{\beta}\right]^{-1} \in[0,1]
$$

390 When the value of $H_{\lambda}$ is closer to zero, the data are believed to have non-observable Poisson

391 homogeneity. Its MLE is

$$
\hat{H}_{\lambda}=\left[1+\frac{1}{\hat{\beta}}\right]^{-1}=\left[1+\frac{s_{\lambda}^{2}}{\bar{\lambda}}\right]^{-1}=\frac{\bar{\lambda}}{\bar{\lambda}+s_{\lambda}^{2}} .
$$

394 The reader is referred to Figure 1 for the configuration of the non-observable Poisson heterogeneity in 395 general.

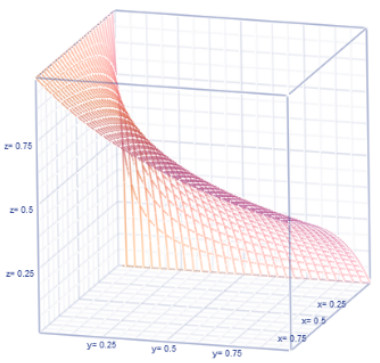

399 Likewise, the observable-heterogeneity is defined below in Definition 2.

401 Definition2. The observable heterogeneity of the randomly sampled Poisson counts, $y_{1}, y_{2}, \ldots . ., y_{n}$ is

402 defined as

403

$$
H_{\bar{y}_{1}}=\left[1+\frac{\operatorname{Var}_{\text {marginal }} E_{\text {posterior }}\left(\lambda \mid \bar{y}_{1}\right)}{E_{\text {marginal }} \operatorname{Var}_{\text {posterior }}\left(\lambda \mid \bar{y}_{1}\right)}\right]^{-1} \in[0,1] .
$$


405 Before we apply the Definition 2, let us recollect that the marginal pdf of the complete sufficient

406 statistic, $\bar{y}_{1}$ is uniform distribution and the posterior distribution is

407

$$
c\left(\lambda \mid \bar{y}_{1}, \alpha, \beta\right)=(1+\beta)^{\left(\alpha+n \bar{y}_{1}\right)}\left[e^{-(1+\beta) \lambda}\right]^{\left(\alpha+n \bar{y}_{1}\right)-1} / \Gamma\left(\alpha+n \bar{y}_{1}\right)
$$

408 with

409

$$
E\left(\lambda \mid \bar{y}_{1}, \alpha, \beta\right)=\frac{\left(\alpha+n \bar{y}_{1}\right)}{(1+\beta)}
$$

410 and

$$
\operatorname{Var}\left(\lambda \mid \bar{y}_{1}, \alpha, \beta\right)=\frac{E\left(\lambda \mid \bar{y}_{1}, \alpha, \beta\right)}{(1+\beta)} .
$$

412 Imposing the Definition 2 and simplifying, we obtain that $H_{\bar{y}_{1}}=\left[1+\frac{(1+\beta)}{6}\right]^{-1}$ whose MLE is

$$
\hat{H}_{\bar{y}_{1}}=\left[1+\frac{(1+\hat{\beta})}{6}\right]^{-1}=\left[1+\frac{\left(1+\frac{\bar{\lambda}}{s_{\lambda}^{2}}\right)}{6}\right]^{-1} \in[0,1] .
$$

414 The reader is referred to Figure 2 for the configuration of the observable Poisson heterogeneity, $\hat{H}_{\bar{y}_{1}}$ in

415 general. When the value of $\hat{H}_{\bar{y}_{1}}$ is closer to zero, the data are interpreted to have observable homogeneity.

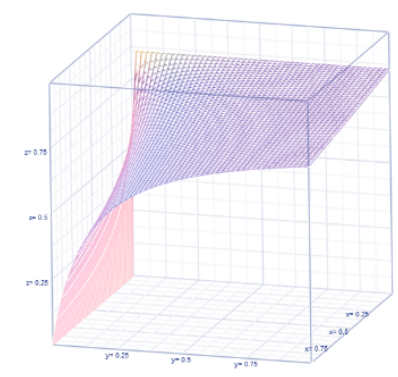


420 Furthermore, the distance, $d\left(y_{1}, \lambda\right)$ between the observable $y_{1}$ of the number of COVID-19 cases and the

421 prevalence rate $\lambda$ could be assessed using the formula

422

423

$$
d\left(y_{1}, \lambda\right)=E_{Y_{1}} E_{\lambda}\left|Y_{1}-\lambda\right|=\sum_{y_{1}=0}^{\infty} \int_{0}^{\infty}\left|Y_{1}-\lambda\right| \operatorname{Pr}\left(y_{1} \mid \lambda\right) c\left(\lambda \mid \bar{y}_{1}, \alpha, \beta\right) d \lambda
$$

424 Realizing that their absolute difference is really $\left|Y_{1}-\lambda\right|=Y_{1}+\lambda-2 \min \left\{Y_{1}, \lambda\right\}$, we obtain after

425 simplifications that

426

$$
d\left(y_{1}, \lambda\right)=\{\beta(1-\beta) \pm 1\}\left(\frac{\alpha}{\{1+\beta\}^{2}}\right)
$$

427 The configuration of the distance, $d\left(y_{1}, \lambda\right)$ between the observable and non-observable in Poisson

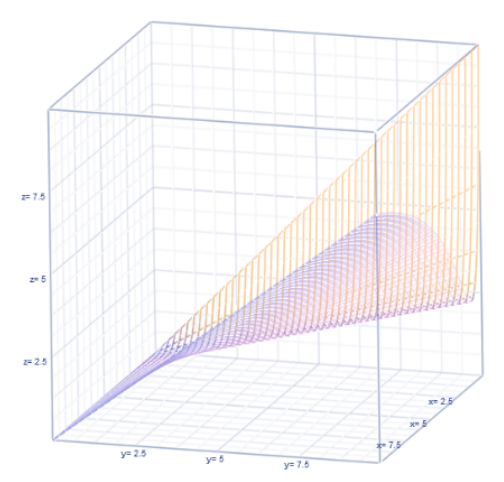

Figure 3. Distance, $d\left(y_{1}, \lambda\right)$ in Poisson.

433 The survival function of the random number, $Y_{1}$ of COVID-19 cases is

$$
S_{Y_{1}}(r \mid \lambda)=\operatorname{Pr}\left(Y_{1} \geq r \mid \lambda\right)=\sum_{i=r}^{\infty} e^{-\lambda} \lambda^{i} / i !=P\left[\chi_{2(r+1) d f}^{2}<2 \lambda\right] ; \lambda>0
$$


435 The hazard rate is a force of mortality. The hazard rate, $h\left(y_{1}\right)$ for the COVID-19 occurrence is

$$
h\left(y_{1}\right)=\frac{\operatorname{Pr}\left(y_{1} \mid \lambda\right)}{S\left(y_{1}+1 \mid \lambda\right)}=\frac{e^{-\lambda} \lambda^{y_{1}}}{y_{1} ! P\left[\chi_{2\left(y_{1}+2\right) d f}^{2}<2 \lambda\right]} ; \lambda>0
$$

439 Does the Poisson chance mechanism keep any a finite memory? For example, the geometric distribution

440 is known to have no memory. What is memory? The memory is really a conditional probability. That is,

$$
\text { memory }=\operatorname{Pr}\left(Y_{1} \geq s \mid y_{1} \geq r\right)=\frac{\operatorname{Pr}\left(Y_{1} \geq r+s\right)}{\operatorname{Pr}\left(Y_{1} \geq r\right)}=\frac{P\left[\chi_{2(r+s+1) d f}^{2}<2 \lambda\right]}{P\left[\chi_{2(r+1) d f}^{2}<2 \lambda\right]} ; \lambda>0,
$$

444 confirming that there is a finite memory in the Poisson mechanism of COVID-19 incidences. To be 445 specific, with $r=0, s=1$ in the above result, the memory between COVID-19 free situation and just one 446 COVID-19 occurrence is revealed in the chance-oriented Poisson mechanism. Such a memory is

$$
\text { memory }_{0 \rightarrow 1}=\frac{P\left[\chi_{4 d f}^{2}<2 \lambda\right]}{P\left[\chi_{2 d f}^{2}<2 \lambda\right]} ; \lambda>0
$$

448 Likewise, the memory between at least one COVID-19 case situation and at least two COVID-19 cases

449 situation is revealed with a substitution of $r=1, s=1$ in the above result and it is

$$
\text { memory }_{1 \rightarrow 2}=\frac{P\left[\chi_{6 d f}^{2}<2 \lambda\right]}{P\left[\chi_{4 d f}^{2}<2 \lambda\right]} ; \lambda>0
$$

451 The odds ratio from the initial memory $_{0 \rightarrow 1}$ to the next memory $y_{1 \rightarrow 2}$ is

$$
O R_{\frac{1 \rightarrow 2}{0 \rightarrow 1}}=\frac{P\left[\chi_{6 d f}^{2}<2 \lambda\right] P\left[\chi_{2 d f}^{2}<2 \lambda\right]}{\left\{P\left[\chi_{4 d f}^{2}<2 \lambda\right]\right\}^{2}}
$$


455 (their values in Table 1). However, the odds for COVID-19 free healthy situation to prevail is

456

457

$$
O d d s_{Y_{1}}=\frac{\operatorname{Pr}\left(Y_{1}=0\right)}{\operatorname{Pr}\left(Y_{1} \geq 1\right)}=\left(e^{\lambda}-1\right)^{-1} ; \lambda>0
$$

459 (their values in Table 1). For details on how the chance for an incidence of a disease to occur from a 460 disease-free scenario changes, the reader is referred to Shanmugam and Radhakrishnan (2011). 


\section{APPENDIX II}

\section{Binomial Heterogeneity: Derivations}

464

Let an indicator random variable, $I_{i}=1$ for a COVID-19 case to be asymptomatic with a probability,

$0<p<1$ and $I_{i}=0$ for the case to be symptomatic with a probability, $0<1-p<1$. Then, for a fixed $y_{1}$,

466 the random variable, $Y_{2}=\sum_{i=1}^{Y_{1}} I_{i}$ follows a binomial probability distribution with parameters $\left(y_{1}, p\right)$.

467 Likewise, for a fixed $y_{1}$, the random variable, $Y_{3}=y_{1}-Y_{2}$ follows a complementary binomial distribution

468 with parameters $\left(y_{1}, 1-p\right)$.That is,

469

$\operatorname{Pr}\left(Y_{2}=y_{2} \mid y_{1}, p\right)=\left(\begin{array}{l}y_{1} \\ y_{2}\end{array}\right) p^{y_{2}}(1-p)^{y_{1}-y_{2}} ; y_{2}=0,1,2, \ldots \ldots, y_{1} ; 0<p<1$

470 and

471

$\operatorname{Pr}\left(Y_{3}=y_{3} \mid y_{1}, p\right)=\left(\begin{array}{l}y_{1} \\ y_{3}\end{array}\right)(1-p)^{y_{3}} p^{y_{1}-y_{3}} ; y_{3}=0,1,2, \ldots \ldots, y_{1} ; 0<1-p<1$

472 with their conditional expected numbers

473

$E\left(Y_{2} \mid y_{1}, p\right)=y_{1} p E\left(Y_{3} \mid y_{1}, 1-p\right)=y_{1}(1-p)=y_{1}-E\left(Y_{2} \mid y_{1}, p\right)$

474 and the conditional variabilities

$475 \operatorname{Var}\left(Y_{2} \mid y_{1}, p\right)=(1-p) E\left(Y_{2} \mid y_{1}, p\right)$,

476 and

$$
\operatorname{Var}\left(Y_{3} \mid y_{1}, 1-p\right)=p E\left(Y_{3} \mid y_{1}, 1-p\right)
$$

The conditional variability of $Y_{2}$ is a percent $(1-p)$ of its expected number $E\left(Y_{2} \mid y_{1}, p\right)$, implying

480 that it exhibits under dispersion. Likewise, the conditional variability of $Y_{3}$ is a percent $(1-p)$ of its 
481 expected number $E\left(Y_{3} \mid y_{1}, p\right)=y_{1}(1-p)$ implying that it also exhibits under dispersion. Together, the

above statements suggest a conditional balance

484 (Stuart and Ord, 2015 for details of the odds concepts). Consequently, we note that

$$
p=\frac{E\left(Y_{2} \mid y_{1}, p\right)}{E\left(Y_{2} \mid y_{1}, p\right)+E\left(Y_{3} \mid y_{1}, 1-p\right)} .
$$

486 Furthermore, we wonder whether the random variables $Y_{2}$ and $Y_{3}$ are correlated? The answer is

487 affirmative. To identify their correlation, notice that

$\operatorname{Var}\left(Y_{3}\right)=E_{Y_{1}} \operatorname{Var}\left(Y_{3} \mid y_{1}\right)+\operatorname{Var}_{Y_{1}} E\left(Y_{3} \mid y_{1}\right)=E_{Y_{1}}\left\{Y_{1} p(1-p)\right\}+\operatorname{Var}_{Y_{1}}\left(Y_{1}\{1-p\}\right)=(1-p) \lambda$

where

$$
\operatorname{Cov}\left(Y_{2}, Y_{3}\right)=E_{Y_{1}} E\left(Y_{2} Y_{3} \mid y_{1}\right)-E_{Y_{1}} E\left(Y_{2} \mid y_{1}\right) E_{Y_{1}} E\left(Y_{3} \mid y_{1}\right)
$$

\section{6}

497

498

$$
\begin{gathered}
E_{Y_{1}} E\left(Y_{2} \mid y_{1}\right)=p \lambda, E_{Y_{1}} E\left(Y_{3} \mid y_{1}\right)=(1-p) \lambda, \\
E_{Y_{1}} E\left(Y_{2} Y_{3} \mid y_{1}\right)=E_{Y_{1}} E_{Y_{2}} E_{Y_{3} \mid Y_{2}, Y_{1}}\left(Y_{2} Y_{3} \mid Y_{1}\right)=E_{Y_{1}} E_{Y_{2} \mid Y_{1}}\left\{Y_{2} Y_{1}(1-p)\right\}=E_{Y_{1}}\left\{p(1-p) Y_{1}^{2}\right\}=p(1-p) \lambda(1+\lambda) .
\end{gathered}
$$

Hence, their correlation is

$$
\rho_{Y_{2}, Y_{3}}=\frac{\operatorname{Cov}\left(Y_{2}, Y_{3}\right)}{\sqrt{\operatorname{Var}\left(Y_{2}\right) \operatorname{Var}\left(Y_{3}\right)}}=\sqrt{p(1-p)}
$$


499 Their expected distance, $d\left(Y_{2}, Y_{3}\right)=E_{Y_{1}} E\left(\left|Y_{2}-Y_{3}\right| \mid Y_{1}\right)$ portrays the drift between the symptomatic

500 observable, $Y_{2}$ and the asymptomatic observable, $Y_{3}$ and it is simplified to this function

$501 d\left(Y_{2}, Y_{3}\right)=|2 p-1| \lambda$ (see Table 3 for their values), due to applying

$$
\left|Y_{2}-Y_{3}\right|=Y_{2}+Y_{3}-2 \min \left\{Y_{2}, Y_{3}\right) \text {. }
$$

for our discussion for asymptomatic COVID-19 cases. The prior average is

$$
\mu_{\text {prior }}=E(p \mid \gamma, \delta)=\frac{\gamma}{(\gamma+\delta)}
$$

511 and the prior variability is

$$
\operatorname{Var}(p \mid \gamma, \delta)=\mu_{\text {prior }}\left(1-\mu_{\text {prior }}\right) /(1+\gamma+\delta)
$$

513 where the parameters $\gamma$ and $\delta$ are hyper-parameters (Rajan and Shanmugam, 2020, for details). We

514 guess that the binomial heterogeneity would involve both hyper parameters. The task for us is how do 515 we construct such heterogeneity? An answer is the following. The posterior distribution

$$
\begin{aligned}
& c\left(p \mid \bar{y}_{1}, \bar{y}_{2}, \gamma, \delta\right)=\operatorname{Pr}\left(\bar{y}_{2} \mid \bar{y}_{1}, p\right) c(p \mid \gamma, \delta) / \int_{-\infty}^{\infty} \operatorname{Pr}\left(\bar{y}_{2} \mid \bar{y}_{1}, p\right) c(p \mid \gamma, \delta) d p \\
& =p^{\gamma+\bar{y}_{2}-1}(1-p)^{\delta+\bar{y}_{1}-\bar{y}_{2}-1} /\left\{\Gamma\left(\gamma+\bar{y}_{2}\right) \Gamma\left(\delta+\bar{y}_{1}-\bar{y}_{2}\right) / \Gamma\left(\gamma+\delta+\bar{y}_{1}\right)\right\}
\end{aligned}
$$


519 would play a key role to construct both the observable and non-observable binomial heterogeneity. With

$\Delta_{p}=p-E(p)$, it is clear that $\int_{-\infty}^{\infty} \Delta_{\lambda} c(p \mid \gamma, \delta) d p=0$

521 The prior variance is

522

$$
\operatorname{Var}(p \mid \gamma, \delta)=\int_{-\infty}^{\infty} \Delta_{\lambda}^{2} c(p \mid \gamma, \delta) d p
$$

Its posterior counterpart

$$
\operatorname{Var}\left(p \mid \bar{y}_{1}, \bar{y}_{2}, \gamma, \delta\right)=\int_{-\infty}^{\infty}\left(p-E\left[p \mid \bar{y}_{1}, \bar{y}_{2}, \gamma, \delta\right)^{2} c(p \mid \gamma, \delta) d p\right.
$$

525 is minimal when the Bayes estimate of non-observable is the posterior mean

$$
p_{\text {Bayes }}=E\left(p \mid \bar{y}_{1}, \bar{y}_{2}, \gamma, \delta\right)
$$

527 where

$528 \quad \mu_{\text {posterior }}=E\left(p \mid \bar{y}_{1}, \bar{y}_{2}, \gamma, \delta\right)=\frac{\left(\gamma+\bar{y}_{2}\right)}{\left(\gamma+\delta+\bar{y}_{1}\right)}$.

529 The posterior variance is

$\operatorname{Var}\left(p \mid \bar{y}_{1}, \bar{y}_{2}, \gamma, \delta\right)=\frac{\mu_{\text {posterior }}\left(1-\mu_{\text {posterior }}\right)}{\left(1+\gamma+\delta+\bar{y}_{1}\right)}$

531 Differentiating the log-likelihood function as

$\ln L\left(n, \bar{y}_{1}, \bar{y}_{2}, p\right)=\bar{y}_{2} \ln p+\left(\bar{y}_{1}-\bar{y}_{2}\right) \ln (1-p)+\sum_{i=1}^{n} \ln \left(\begin{array}{c}y_{i} \\ y_{2, i}\end{array}\right)$

533 with respect to the non-observable parameter, $p$, setting it equal to zero and solving it, we obtain the

$534 \quad$ MLE and it is $\hat{p}_{m l e}=\frac{\bar{y}_{2}}{\bar{y}_{1}}$ It is known that

$$
E_{\text {prior }} E_{\text {likelihood }}\left(\bar{y}_{2} \mid \bar{y}_{1}, p\right)=E\left(\bar{y}_{2}\right)
$$


$537 \operatorname{Var}\left(\bar{y}_{2}\right)=E_{\text {prior }} \operatorname{Var}_{\text {likelihood }}\left(\bar{y}_{2} \mid \bar{y}_{1}, p\right)+\operatorname{Var}_{\text {prior }} E_{\text {likelihood }}\left(\bar{y}_{2} \mid \bar{y}_{1}, p\right)$.

539 Hence, we define the non-observable binomial heterogeneity below in Definition 3.

Definition 3. The non-observable binomial heterogeneity is defined as

$$
H_{y_{1}, p}=\left[1+\frac{\text { Var }_{\text {prior }} E_{\text {likelihood }}\left(\bar{y}_{2} \mid \bar{y}_{1}, p\right)}{E_{\text {prior }} \operatorname{Var}_{\text {likelihood }}\left(\bar{y}_{2} \mid \bar{y}_{1}, p\right)}\right]^{-1} \in[0,1]
$$

545 Following the Definition 3, we obtain the non-observable heterogeneity of the COVID-19's asymptotic

$H_{y_{1}, \gamma, \delta}=\left[1+y_{1} \frac{\operatorname{Var}_{\text {prior }}(p)}{E_{\text {prior }}(p\{1-p\})}\right]^{-1}=\left[1+\frac{y_{1}}{(\gamma+\delta)(1+\gamma+\delta)}\right]^{-1} \in[0,1]$

548 When the value of $H_{y_{1}, \gamma, \delta}$ is closer to zero, the data are interpreted to have non-observable binomial

549 homogeneity. Substituting the MLEs

550

$\hat{\gamma}=\bar{p}\left\{\frac{\bar{p}(1-\bar{p})}{s_{p}^{2}}-1\right\}$ and $\hat{\delta}=\frac{(1-\bar{p}) \hat{\gamma}}{\bar{p}}$

551 we obtain its MLE

552

$$
\hat{H}_{y_{1}, \gamma, \delta}=\left[1+\frac{y_{1}\left(s_{p}^{2}\right)^{2}}{\left(\left|\bar{p}(1-\bar{p})-s_{p}^{2}\right|\right) \bar{p}(1-\bar{p})}\right]^{-1} \in[0,1]
$$

553 Likewise, the observable-heterogeneity of the binomial distribution of $y_{2}$ is defined below in Definition 
555 Definition 4. The observable heterogeneity of the binomial counts, $y_{2, i}, i=1,2, \ldots, y_{1}$ (in terms of the

556 complete sufficient statistic $\bar{y}_{2}$ ) is defined as

$H_{y_{2}}=\left[1+\frac{\operatorname{Var}_{\text {marginal }} E_{\text {posterior }}\left(p \mid \bar{y}_{1}\right)}{E_{\text {marginal }} \operatorname{Var}_{\text {posterior }}\left(p \mid \bar{y}_{1}\right)}\right]^{-1} \in[0,1]$

558 Before we apply Definition 4, remember that the marginal pdf of the complete sufficient statistic, $\bar{y}_{2}$ is

559 the beta-binomial distribution,

560

$\operatorname{Pr}\left(\bar{Y}_{2}\right)=\left(\begin{array}{l}\bar{y}_{1} \\ \bar{y}_{2}\end{array}\right) \frac{\Gamma\left(\gamma+\bar{y}_{2}\right) \Gamma\left(\delta+\bar{y}_{1}-\bar{y}_{2}\right)}{\left.\Gamma\left([\gamma+\delta]+\bar{y}_{1}\right)\right\}}$

561 and the posterior distribution is beta. With the notation $B(a, b)=\frac{\Gamma(a) \Gamma(b)}{\Gamma(a+b)}$, we note that the probability

562 mass function of the beta-binomial distribution is

563

$$
\operatorname{Pr}\left(y_{2}\right)=\left(\begin{array}{l}
y_{1} \\
y_{2}
\end{array}\right) B\left(\gamma+y_{2}, \delta+y_{1}-y_{2}\right) / B(\gamma, \delta) ; y_{2}=0,1,2 \ldots, y_{1} ; \gamma, \delta>0
$$

564 That is, the posterior probability density function is

$$
c\left(p \mid \bar{y}_{1}, \bar{y}_{2}, \gamma, \delta\right)=\frac{\left.\Gamma\left(\gamma+\delta+\bar{y}_{1}\right)\right\}}{\Gamma\left(\gamma+\bar{y}_{2}\right) \Gamma\left(\delta+\bar{y}_{1}-\bar{y}_{2}\right)} p^{\gamma+\bar{y}_{2}-1}(1-p)^{\delta+\bar{y}_{1}-\bar{y}_{2}-1}
$$

566 with

567

$$
E\left(p \mid \bar{y}_{1}, \bar{y}_{2}, \gamma, \delta\right)=\frac{\left(\gamma+\bar{y}_{2}\right)}{\left.\left([\gamma+\delta]+\bar{y}_{1}\right)\right)}
$$

568 and

569

$$
\operatorname{Var}\left(p \mid \bar{y}_{1}, \bar{y}_{2}, \gamma, \delta\right)=\frac{\left(\gamma+\bar{y}_{2}\right)\left(\delta+\bar{y}_{1}-\bar{y}_{2}\right)}{\left([\gamma+\delta]+\bar{y}_{1}\right)\left(1+[\gamma+\delta]+\bar{y}_{1}\right)}
$$

570 Now applying Definition 4, we obtain an expression for the observable binomial heterogeneity 


$$
\left.H_{y_{2} \mid y_{1}}=\left[1+\frac{\operatorname{Var}_{\text {marginal }}\left\{\frac{\left(\gamma+y_{2}\right)}{\left(\gamma+\delta+y_{1}\right)}\right\}}{E_{\text {marginal }}\left\{\frac{\left(\gamma+y_{2}\right)\left(\delta+y_{1}-y_{2}\right)}{\left(\gamma+\delta+y_{1}\right)\left(1+\gamma+\delta+y_{1}\right)}\right.}\right\}\right]^{-1} \approx\left[1+\left(\frac{\delta}{\delta+\gamma}\right)\left(\frac{y_{1}}{y_{1}+\delta}\right)\right]^{-1} \in[0,1],
$$

573 whose estimate is

574

$$
H_{y_{2} \mid y_{1}} \approx\left[1+\bar{p}\left(\frac{y_{1} s_{p}^{2}}{y_{1} s_{p}^{2}+\{1-\bar{p}\}\left|\bar{p}(1-\bar{p})-s_{p}^{2}\right|}\right)\right]^{-1},
$$

Because

$$
\hat{\gamma} \approx \bar{p}\left\{\frac{\bar{p}(1-\bar{p})}{s_{p}^{2}}-1\right\} \quad \text { and } \quad \hat{\delta} \approx \frac{\hat{\gamma}(1-\bar{p})}{\bar{p}}
$$

577 When the value of $\hat{H}_{y_{2} \mid y_{1}}$ is closer to zero, the data are considered to have observable binomial

578 homogeneity. Also, the distance, $d\left(y_{2}, p\right)$ between the observable $y_{2}$ of the number of asymptomatic

579 COVID-19 cases and its proportion, $p$ could be assessed using the formula

580

$$
d\left(y_{2}, p\right)=E_{Y_{2}} E_{p}\left|Y_{2}-p\right|=\sum_{y_{2}=0}^{y_{1}} \int_{0}^{\infty}\left|Y_{2}-p\right| \operatorname{Pr}\left(y_{2} \mid p\right) c\left(p \mid y_{1}, y_{2}, \gamma, \delta\right) d p .
$$

581 Realizing that the absolute difference, $\left|Y_{2}-p\right|=Y_{2}+p-2 \min \left\{Y_{2}, p\right\}$, we obtain after simplifications that

$$
d\left(y_{2}, p\right)=\left|y_{1}-1\right|\left(\frac{\gamma}{\gamma+\delta}\right) .
$$

584 have to do is change $p$ to $(1-p)$, change $y_{2}$ to $y_{3}$, along with changing $\gamma$ to $\delta$ and go through the process

$$
H_{y_{1}, \delta, \gamma}=\left[1+y_{1} \frac{\text { Var }_{\text {prior }}(1-p)}{E_{\text {prior }}(p\{1-p\})}\right]^{-1}=\left[1+\frac{y_{1}}{(\gamma+\delta)(1+\gamma+\delta)}\right]^{-1} \in[0,1] \text {. }
$$

587 The observable binomial heterogeneity for the symptomatic cases is 


$$
H_{y_{3} \mid y_{1}} \approx\left[1+\left(\frac{\gamma}{\delta+\gamma}\right)\left(\frac{y_{1}}{y_{1}+\gamma}\right)\right]^{-1} \in[0,1]
$$

whose MLE is

$$
\hat{H}_{y_{3} \mid y_{1}} \approx\left[1+\bar{p}\left(\frac{y_{1} s_{p}^{2}}{y_{1} s_{p}^{2}+\bar{p}\left|\bar{p}(1-\bar{p})-s_{p}^{2}\right|}\right)\right]^{-1}
$$

591 which is interestingly not the same as $\hat{H}_{y_{2} \mid y_{1}}$. Also, the distance, $d\left(y_{3}, 1-p\right)$ between the observable $y_{3}$ of

592 the number of asymptomatic COVID-19's symptomatic cases and the proportion, $1-p$ could be assessed

593 using the formula

$$
d\left(y_{3}, 1-p\right)=E_{Y_{2}} E_{p}\left|Y_{2}-(1-p)\right|=\sum_{y_{3}=0}^{y_{1}} \int_{0}^{\infty}\left|Y_{3}-(1-p)\right| \operatorname{Pr}\left(y_{3} \mid 1-p\right) c\left(1-p \mid y_{1}, y_{2}, \gamma, \delta\right) d(1-p)
$$

and it is after simplifications that

$$
d\left(y_{3}, p\right)=\left|y_{1}-1\right|\left(\frac{\delta}{\gamma+\delta}\right) .
$$

$$
S_{Y_{2}}\left(r, p \mid y_{1}\right)=\operatorname{Pr}\left(Y_{2} \geq r \mid y_{1}\right)=\sum_{i=r}^{\infty} \frac{y_{1} !}{i !\left(y_{1}-i\right) !} p^{i}(1-p)^{y_{1}-i}=P\left[F_{\left(2 r, 2\left[y_{1}-r+1\right]\right) d f} \leq \frac{y_{1} p\left(y_{1}-r+1\right)}{(1-p) r}\right] ; 0<p<1 .
$$

602 The hazard rate, $h(y)$ of the binomial distribution for the asymptomatic cases is

$$
h\left(y_{2}\right)=\frac{\operatorname{Pr}\left(y_{2} \mid p\right)}{S\left(y_{2}+1 \mid p\right)}=\frac{y_{1} !\left\{\frac{p}{1-p}\right\}^{y_{2}}\{1-p\}^{y_{1}}}{y_{2} !\left(y_{1}-y_{2}\right) ! P\left[F_{\left(2 r, 2\left[y_{1}-r+1\right]\right) d f} \leq \frac{y_{1} p\left(y_{1}-r+1\right)}{\{1-p\} r}\right]} ; 0<p<1 .
$$

604 The binomial distribution has a finite memory 
$\operatorname{Pr}\left(Y_{2} \geq s \mid y_{2} \geq r\right)=\frac{\operatorname{Pr}\left(Y_{2} \geq r+s\right)}{\operatorname{Pr}\left(Y_{2} \geq r\right)}=\frac{P\left[F_{\left(2 r, 2\left[y_{1}-(r+s)+1\right]\right) d f} \leq \frac{y_{1} p\left(y_{1}-(r+s)+1\right)}{\{1-p\}(r+s)}\right]}{P\left[F_{\left(2 r, 2\left[y_{1}-r+1\right]\right) d f} \leq \frac{y_{1} p\left(y_{1}-r+1\right)}{\{1-p\} r}\right]}$.

606 confirming that the usual binomial distribution does possess a finite memory. The conditional odds, for a 607 fixed $y_{1}$, for safe asymptomatic symptom are

608

$O d d s_{Y_{2} \mid y_{1}}=\frac{\operatorname{Pr}\left(Y_{2}=0\right)}{\operatorname{Pr}\left(Y_{2} \geq 1\right)}=(1-p)^{y_{1}}\left\{1-(1-p)^{y_{1}}\right\}^{-1} \approx(1-p)^{y_{1}}\left\{1+(1-p)^{y_{1}}\right\}$

609 The unconditional odds for safe asymptotic symptom are

$$
\operatorname{odds}_{Y_{2}} \approx \sum_{y_{1}=0}^{\infty} O d d s_{Y_{2} \mid y_{1}} \operatorname{Pr}\left[Y_{1}=y_{1} \mid \lambda\right] \approx \sum_{y_{1}=0}^{\infty}(1-p)^{y_{1}}\left\{1+(1-p)^{y_{1}}\right\} e^{-\lambda} \lambda^{y_{1}} / y_{1} ! \approx e^{-p \lambda}\left\{1+e^{-p(1-p) \lambda}\right\}
$$

612 The reader is referred to Figure 4 for the configuration of the odds in asymptotic COVID-19 occurrences

613 in general.

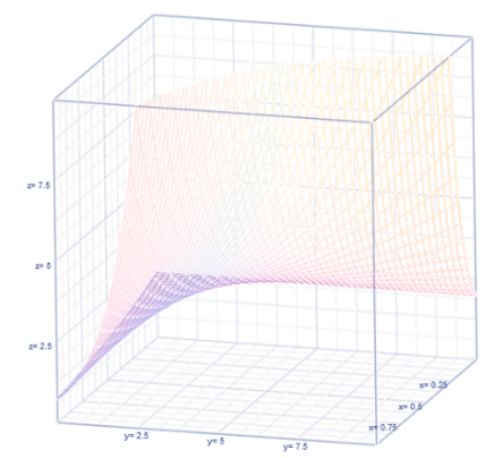

617 Recall that $S_{Y_{2}}\left(1, p \mid y_{1}\right)=\operatorname{Pr}\left(Y_{2} \geq 1 \mid y_{1}\right)$ is the likelihood for the existence of asymptomatic presentation of

618 COVID-19 in the ship. The hazard in that situation (that is, with $r=1$ ) is 


$$
h_{Y_{2}}\left(1 \mid y_{1}, p\right)=1-\frac{P\left[F_{\left(4,2\left[y_{1}-1\right]\right) d f} \leq \frac{y_{1} \hat{p}_{m l e}\left(y_{1}-1\right)}{2\left(1-\hat{p}_{m l e}\right)}\right]}{P\left[F_{\left(4,2 y_{1}\right) d f} \leq \frac{y_{1}^{2} \hat{p}_{m l e}}{\left(1-\hat{p}_{m l e}\right)}\right]},
$$

621 where $\hat{p}_{m l e}=\bar{y}_{2}$. A popular statistical concept in the business world (Khokhlov, 2016 for details), Tail

Value at Risk (Tar) is

623

$$
T \operatorname{VaR}_{Y_{2}}=E\left[Y_{2} \mid Y_{2} \geq 1, p, y_{1}\right] \approx 1+\frac{y_{1}(1-p)}{p^{2} P\left[F_{\left(2 r, 2 y_{1}\right) d f} \leq \frac{y_{1}^{2} p}{(1-p)}\right]}
$$

624 Similarly, all the Bayesian results for the binomial random variable, $y_{3}$ are easily derivable by

625 interchanging $\gamma$ and $\delta$ in all above expressions. The survival function of the random number, $Y_{3}$ with

626 symptomatic symptoms is

$S_{Y_{3}}\left(r, p \mid y_{1}\right)=\operatorname{Pr}\left(Y_{3} \geq r \mid y_{1}\right)=\sum_{i=r}^{\infty} \frac{y_{1} !}{i !\left(y_{1}-i\right) !}(1-p)^{i} p^{y_{1}-i}=P\left[F_{\left(2 r, 2\left[y_{1}-r+1\right]\right) d f} \leq \frac{y_{1}(1-p)\left(y_{1}-r+1\right)}{p r}\right] ; 0<p<1$.

629 The hazard rate, $h(y)$ for the symptomatic sign is

$$
h\left(y_{3}\right)=\frac{\operatorname{Pr}\left(y_{3} \mid p\right)}{S\left(y_{3}+1 \mid p\right)}=\frac{y_{1} !\left\{\frac{1-p}{p}\right\}^{y_{3}} p^{y_{1}}}{y_{3} !\left(y_{1}-y_{2}\right) ! P\left[F_{\left(2 r, 2\left[y_{1}-r+1\right]\right) d f} \leq \frac{y_{1}(1-p)\left(y_{1}-r+1\right)}{p r}\right]} ; 0<p<1 .
$$

632 The binomial distribution of those with symptomatic signs has a finite memory

$$
\operatorname{Pr}\left(Y_{3} \geq s \mid y_{3} \geq r\right)=\frac{\operatorname{Pr}\left(Y_{3} \geq r+s\right)}{\operatorname{Pr}\left(Y_{3} \geq r\right)}=\frac{P\left[F_{\left(2 r, 2\left[y_{1}-(r+s)+1\right]\right) d f} \leq \frac{y_{1}(1-p)\left(y_{1}-(r+s)+1\right)}{p(r+s)}\right]}{P\left[F_{\left(2 r, 2\left[y_{1}-r+1\right]\right) d f} \leq \frac{y_{1}(1-p)\left(y_{1}-r+1\right)}{p r}\right]},
$$


635 confirming that the usual binomial probability trend of those with symptomatic signs does possess a

636 finite memory. The conditional odds, for a fixed $y_{1}$, for safe symptomatic symptom are

$\operatorname{Odds}_{Y_{3} \mid y_{1}}=\frac{\operatorname{Pr}\left(Y_{3}=0\right)}{\operatorname{Pr}\left(Y_{3} \geq 1\right)}=p^{y_{1}}\left\{1-p^{y_{1}}\right\}^{-1} \approx p^{y_{1}}\left\{1+p^{y_{1}}\right\}$

638 The unconditional odds for safe symptomatic symptom are

$\operatorname{odds}_{Y_{3}} \approx \sum_{y_{1}=0}^{\infty} \operatorname{Odds}_{y_{1}} \operatorname{Pr}\left[Y_{1}=y_{1} \mid \lambda\right] \approx \sum_{y_{1}=0}^{\infty} p^{y_{1}}\left\{1+p^{y_{1}}\right\} e^{-\lambda} \lambda^{y_{1}} / y_{1} ! \approx e^{-(1-p) \lambda}\left\{1+e^{-p(1-p) \lambda}\right\}$

640 A comparison of $o d d s_{Y_{2}}$ and $o d d s_{Y_{3}}$ suggests the odds ratio,

$641 \quad O R_{Y_{3} / Y_{2}}=\frac{o d d s_{Y_{3}}}{o d d s_{Y_{2}}}=e^{-(1-2 p) \lambda}$.

642 See Figure 5 for the configuration of the isomorphic factor, $e^{-(1-2 p) \lambda}$.

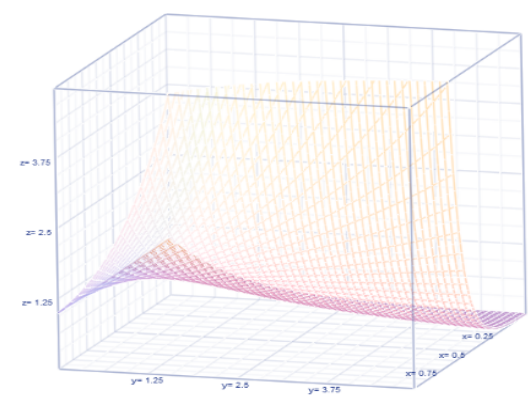

646 Recall that $S_{Y_{3}}\left(1, p \mid y_{1}\right)=\operatorname{Pr}\left(Y_{3} \geq 1 \mid y_{1}\right)$ is the chance for the existence of symptomatic symptom of

647 COVID-19. The hazard in that situation (that is, with $r=1$ ) is 


$$
648 h_{Y_{3}}\left(1 \mid y_{1}, p\right)=1-\frac{P\left[F_{\left(4,2\left[y_{1}-1\right]\right) d f} \leq \frac{y_{1}\left(1-\hat{p}_{m l e}\right)\left(y_{1}-1\right)}{2 \hat{p}_{m l e}}\right]}{P\left[F_{\left(4,2 y_{1}\right) d f} \leq \frac{y_{1}^{2}\left(1-\hat{p}_{m l e}\right)}{\hat{p}_{m l e}}\right]},
$$

649 where $\hat{p}_{m l e}=\bar{y}_{2}$. The Tail Value at Risk (TVaR) is

$$
\operatorname{TVaR}_{Y_{3}}=E\left[Y_{3} \mid Y_{3} \geq 1, p, y_{1}\right] \approx 1+\frac{y_{1} p}{(1-p)^{2} P\left[F_{\left(2 r, 2 y_{1}\right) d f} \leq \frac{y_{1}^{2}(1-p)}{p}\right]}
$$

